\title{
On the Achievable Region for Multiple Description Source Codes on Gaussian Sources
}

\author{
Hanying Feng ${ }^{1}$ \\ Teradyne Inc. \\ Agoura Hills, CA 91301 \\ email: fhy@z.caltech.edu
}

\begin{abstract}
We demonstrate inconsistencies in prior results on the achievable region for multiple description (MD) source codes on iid Gaussian sources with the squared error distortion measure. We then describe the complete region.
\end{abstract}

\section{Previous Results}

Suppose that an MD code for source $X \sim \mathcal{N}\left(0, \sigma^{2}\right)$ has two side reconstructions $Y_{1}$ and $Y_{2}$ with expected distortions $D_{1}$ and $D_{2}$, respectively, and a joint reconstruction $Y_{0}$ with expected distortion $D_{0}$. Prior authors use two distinct approaches for building the reproductions needed to apply El Gamal and Cover's 2DSC-achievable ratedistortion result [1]. To discuss those results, we define

$$
\begin{aligned}
& R(D)=\frac{1}{2} \log \frac{\sigma^{2}}{D} \\
& D_{L}\left(D_{1}, D_{2}\right)=D_{1}+D_{2}-\sigma^{2} \\
& D_{H}\left(D_{1}, D_{2}\right)=\left(\frac{1}{D_{1}}+\frac{1}{D_{2}}-\frac{1}{\sigma^{2}}\right)^{-1} \\
& \mathcal{D}_{1}=\left\{\left(D_{0}, D_{1}, D_{2}\right): D_{0} \in\left[0, D_{L}\left(D_{1}, D_{2}\right)\right)\right\} \\
& \mathcal{D}_{2}=\left\{\left(D_{0}, D_{1}, D_{2}\right): D_{0} \in\left(D_{L}\left(D_{1}, D_{2}\right), D_{H}\left(D_{1}, D_{2}\right)\right)\right\} \\
& \mathcal{D}_{3}=\left\{\left(D_{0}, D_{1}, D_{2}\right): D_{0} \in\left[D_{H}\left(D_{1}, D_{2}\right), \infty\right)\right\} \\
& L_{G 0}=\frac{1}{2} \log \frac{\left(\sigma^{2}-D_{0}\right)^{2}}{\left(\sigma^{2}-D_{0}\right)^{2}-(A-B)^{2}} \\
& L_{G 12}=\frac{1}{2} \log \frac{\left(\sigma^{2}-D_{0}\right)^{2} D_{1} D_{2}}{\left(\sigma^{2}-D_{0}\right)^{2} D_{1} D_{2}-\left(\sigma^{2} B-D_{0} A\right)^{2}},
\end{aligned}
$$

where $A=\sqrt{\left(\sigma^{2}-D_{1}\right)\left(\sigma^{2}-D_{2}\right)}$ and $B=\sqrt{\left(D_{1}-D_{0}\right)\left(D_{2}-D_{0}\right)}$.

The approach used in [1], which we call the "joint decoder first" approach, sets up $X \rightarrow Y_{0} \rightarrow\left(Y_{1}, Y_{2}\right)$ as a Markov chain. The resulting achievable region is

$$
R_{1} \geq R\left(D_{1}\right), R_{2} \geq R\left(D_{2}\right), R_{1}+R_{2} \geq R\left(D_{0}\right)+L_{0}
$$

where $L_{0}=0$ in $\mathcal{D}_{1}$ and $L_{0}=L_{G 0}$ in $\mathcal{D}_{2} \cup \mathcal{D}_{3}$.

The approach of $[2,3]$, which we call the "side decoders first" approach, sets up $X \rightarrow\left(Y_{1}, Y_{2}\right) \rightarrow Y_{0}$ as a Markov chain. The resulting achievable region is

$$
\begin{aligned}
& R_{1} \geq R\left(D_{1}\right), \quad R_{2} \geq R\left(D_{2}\right), \\
& R_{1}+R_{2} \geq R\left(D_{1}\right)+R\left(D_{2}\right)+L_{12},
\end{aligned}
$$

where $L_{12}=\frac{1}{2} \log \frac{D_{1} D_{2}}{\sigma^{2}\left(D_{1}+D_{2}-\sigma^{2}\right)}$ in $\mathcal{D}_{1}, L_{12}=L_{G 12}$ in $\mathcal{D}_{2}$, and $L_{12}=0$ in $\mathcal{D}_{3}$.

While the authors claim agreement with each other and with Ozarow's converse [2], the results actually differ for some $\left(D_{0}, D_{1}, D_{2}\right)$ values. In Section II, we discuss the

\footnotetext{
${ }^{1}$ This material is based upon work partially supported by NSF Grant No. CCR-0220039 and Caltech's Lee Center for Advanced Networking.
}

\author{
Michelle Effros \\ Department of Electrical Engineering \\ California Institute of Technology \\ Pasadena, CA 91125 \\ email: effros@caltech.edu
}

derivation for the complete achievable region, pointing out the discrepancies with prior characterizations. The given achievable region is tight for all $\left(D_{0}, D_{1}, D_{2}\right)$.

\section{RESULTS}

We treat regions $\mathcal{D}_{1}, \mathcal{D}_{2}$, and $\mathcal{D}_{3}$ separately.

Theorem 1 In $\mathcal{D}_{1}$, the achievable region is

$$
R_{1} \geq R\left(D_{1}\right), \quad R_{2} \geq R\left(D_{2}\right), \quad R_{1}+R_{2} \geq R\left(D_{0}\right) . \text { (3) }
$$

Theorem 1, which relies on the joint decoder first approach, differs from (2) in region $\mathcal{D}_{1}$. The side decoders first approach gives the smaller achievable region $R_{1} \geq R\left(D_{1}\right), R_{2} \geq R\left(D_{2}\right)$, and $R_{1}+R_{2} \geq R\left(D_{1}\right)+$ $R\left(D_{2}\right)+L_{G 12}$ in $\mathcal{D}_{1}$. This implies that the derivation in [3] that uses this approach to get a Shannon type inner bound in $\mathcal{D}_{1}$ is problematic.

Theorem 2 In $\mathcal{D}_{2}$, the achievable region is

$$
\begin{aligned}
R_{1} & \geq R\left(D_{1}\right), \quad R_{2} \geq R\left(D_{2}\right), \\
R_{1}+R_{2} & \geq R\left(D_{0}\right)+L_{G 0}=R\left(D_{1}\right)+R\left(D_{2}\right)+L_{G 12} .
\end{aligned}
$$

Theorem 2 can be proven with either approach; therefore it agrees with both (1) and (2) in region $\mathcal{D}_{2}$.

Theorem 3 In $\mathcal{D}_{3}$, the achievable region is

$$
R_{1} \geq R\left(D_{1}\right), R_{2} \geq R\left(D_{2}\right) .
$$

Theorem 3 differs from (1) in $\mathcal{D}_{3}$, where the joint decoder first approach additionally forces $R_{1}+R_{2} \geq$ $R\left(D_{0}\right)+L_{G 0}$.

\section{ConClusions}

Neither the joint decoder first nor the side decoders first approach is sufficient to find the complete achievable region. The no excess rate sum case given by (3) is achievable only in $\mathcal{D}_{1}$ using the joint decoder first approach. The no excess marginal rate case given by (4) is achievable only in $\mathcal{D}_{3}$ using the side decoders first approach. Both approaches yield the identical optimal result in $\mathcal{D}_{2}$.

\section{REFERENCES}

[1] A. A. El Gamal and T. M. Cover, "Achievable Rates for Multiple Descriptions," IEEE Trans.-IT, 28:851-857, Nov. 1982.

[2] L. Ozarow, "On a Source-coding Problem with Two Channels and Three Receivers," Bell Syst. Tech. J., 59:19091921, Dec. 1980.

[3] R. Zamir, "Gaussian codes and shannon bounds for multiple descriptions", IEEE Trans.-IT, 45:2629-2636, Nov. 1999. 